\title{
Atomistic modelling of the hypervelocity dynamics of shock-compressed graphite and impacted graphene armours
}

\author{
Stefano Signetti ${ }^{\mathrm{a}}$, Keonwook Kang ${ }^{\mathrm{b}, *}$, Nicola M. Pugno ${ }^{\mathrm{c}, \mathrm{d}, \mathrm{e}}$, Seunghwa Ryu ${ }^{\mathrm{a}, *}$ \\ ${ }^{a}$ Department of Mechanical Engineering, Korea Advanced Institute of Science and Technology (KAIST), \\ 291 Daehak-ro, Yuseong-gu, Daejeon 34141, Republic of Korea \\ ${ }^{b}$ Department of Mechanical Engineering, Yonsei University, \\ 50 Yonsei-ro, Sodaemun-gu, Seoul 03722, Republic of Korea \\ ${ }^{c}$ Laboratory of Bio-Inspired and Graphene Nanomechanics, \\ Department of Civil, Environmental and Mechanical Engineering, University of Trento, \\ via Mesiano 77, I-38123 Trento, Italy \\ ${ }^{d}$ School of Engineering and Materials Science, Queen Mary University of London, \\ Mile End Road, E1 4NS London, United Kingdom \\ ${ }^{e}$ Ket-Lab, Edoardo Amaldi Foundation, via del Politecnico snc, I-00133 Roma, Italy
}

\begin{abstract}
We present a molecular dynamics (MD) simulation study on the hypervelocity dynamics of shock compressed graphite -up to hundreds of gigapascals- and impacted multilayer graphene armours by employing the AIREBO-M potential. The Morse-type non-singular intermolecular interaction allows the usage of relatively large integration timesteps for simulating materials' response at such high strain-rate. The MD simulation results are in good agreement with the shock Hugoniot curves and with graphite-to-diamond transition obtained from both density functional theory (DFT) and experiments available in literature. We then show that thermodynamic properties of graphite from MD calculations can be used as input for a reliable equation of state to be employed in continuum simulations. Finally, we find that the size-scaling of the hypervelocity impact properties of graphene armours matches well with the DFT results and theoretical predictions of earlier studies. Our results open a concrete possibility towards accurate and fast multiscale simulation from atomistic to continuum level of shock propagation, shock-induced phase transformation, and dynamic fracture in large or hierarchical carbon systems, such as graphene-based foams and nanocomposites.
\end{abstract}

\footnotetext{
${ }^{*}$ Corresponding author.

Email addresses: kwkang@yonsei.ac.kr (Keonwook Kang), ryush@kaist.ac.kr (Seunghwa Ryu)
} 


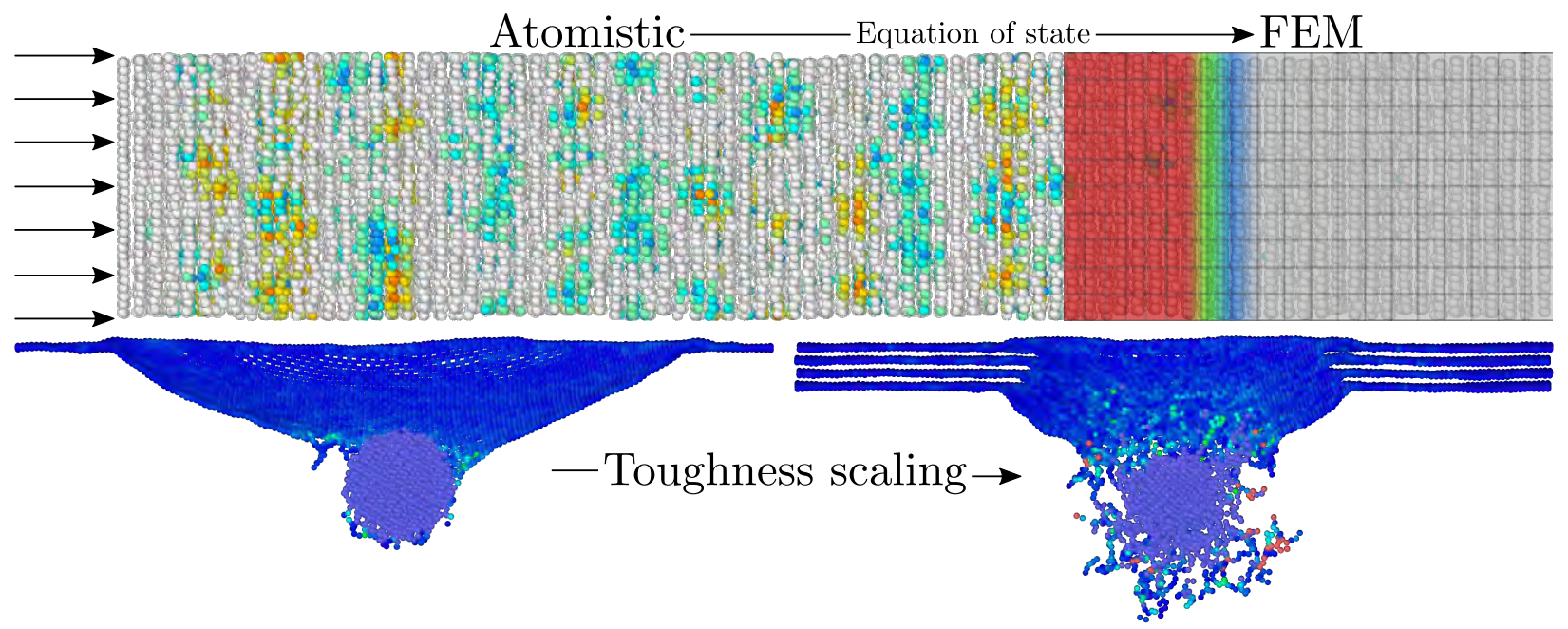

Keywords: Atomistic modelling, Hypervelocity shock, Interatomic forces, Equation of state, Carbon foams, Graphene nanocomposites

\section{Introduction}

The study of the equation of state (EOS) of carbon at high pressure is of increasing interest since the advent of graphene, whose exceptional mechanical properties make it the ideal candidate to fabricate lightweight armours with extremely high specific toughness, either exploited alone [1] or as reinforcement in layered nanocomposites [2]. However, the behaviour of carbon at extremely high pressures is likewise of paramount importance in other fields of materials science and physics, e.g. in planetary sciences and astrophysics: we mention the formation of carbon allotropes from geological and meteorite impact processes [3, 4] or the understanding of the nature of $\mathrm{C}$ in the interior and atmosphere of giant planets, such as Uranus and Neptune (pressures up to $600 \mathrm{GPa}$ in the core) [5, 6].

Numerous studies of the carbon phase diagram through isotropic and uniaxial compression experiments and simulations have been performed. Most of these studies focus on relatively moderate compression regimes $(<150 \mathrm{GPa})$, where graphite undergoes a phase transition toward diamond (G/D transition) [7/9]. However, the above mentioned applications require also investigation at pressures up to several hundreds of gigapascals, which up to now remains still few and limited, mainly to diamond [10-16], due to experimental difficulties 
in producing controlled shock loads and monitoring of state variables. The experimental data display also some variability in the observed properties (i.e. pressure, temperature, density) which may relate to the amount of each mechanism involved, with the diffusion process supposedly yielding substantially lower resistance to diamond nucleation, which is also affected by sample geometrical characteristics. Overall, the experimental data suggest that the Hugoniot curve and the resulting G/D transition process depend substantially on the degree of crystallinity, purity, porosity and defects, and orientation of the shocked sample [8, 17. Hence, reliable computational models can complement experiments for deepening our understanding of materials behaviour under such high pressures and the cause of variability of material parameters.

Molecular dynamics (MD) simulations of the high pressure properties of graphite are scarce due to the lack of interaction models capable of describing with sufficient accuracy its subtle combination of covalent and non-bonded interactions. Recently, Pineau [18] showed that the LCBOPII potential [19], which includes long-range interactions to account for non-bonded interactions and medium-range contribution fitted to $a b$ initio calculations of the dissociation energy curves of simple, double, and triple bonds, can accurately reproduce the DFT predicted G/D barrier and transition to liquid carbon but still overestimates the material stiffness (pressure Hugoniot) at very large shock pressures. Resulting errors in computing material pressure, density, and particle velocity, namely its shock impedance, affects the tracking of wave propagation and, consequently, prediction of spall fracture in pristine graphite and, therefore, in resulting nanocomposites [20]. Indeed, we have recently shown [1] that the most commonly used MD carbon potentials [21, 22] cannot accurately predict the scaling of the absorbed energy, i.e. the absorbed energy per areal density, in multilayer graphene armours subjected to the hypervelocity impact of a traveling mass, yielding at the nanoscale to significant lower estimates and suboptimal scaling with respect to what predicted by DFT and theoretical calculations.

The classical AIREBO potential by Stuart et al. [23], which is widely used to model the deformation and fracture of graphitic structure -ranging from few layer graphene to carbon nanotubes and foams- also accounts, with respect to covalent REBO2 [24], for non-bonded 
intermolecular interactions for hydrocarbon compounds via a set of smoothly truncated 6-12 Lennard-Jones (LJ) potentials in the form:

$$
\Pi_{\mathrm{LJ}}(r)=4 \epsilon\left[\left(\frac{\sigma}{r}\right)^{12}-\left(\frac{\sigma}{r}\right)^{6}\right]
$$

where $r$ is the distance between two pair atoms, $\sigma$ is the inter-particle distance at which the potential is zero, and $\epsilon$ is the corresponding bond energy. The potential is smoothly set to zero by a third-order spline at a long-range cutoff value of $3 \sigma$. For separations less than the equilibrium van der Waals distance $h=\sqrt[6]{2} \sigma$, similar splines reduce the strength of intermolecular repulsions if it is favorable for a pair of atoms to bond chemically. When covalent bonding is unfavorable, the two atoms interact with the full LJ [23]. Repulsive $r^{12}$ power law in the LJ, which has no physical basis, causes the intermolecular interaction force to rise rapidly as molecular separation decreases, such as occurring under high shock pressures. This may yield to anomalously high repulsive force during molecular dynamics simulations with a finite time step, which often results in unphysical acceleration of atoms and increase of instantaneous temperature. Adoption of smaller timesteps $(<\mathrm{fs})$ may be not sufficient to mitigate the problem and heavily impacts on the computational cost anyhow. To improve the accuracy of the interaction model at high pressures O'Connor et al. 25] replaced LJ potentials with a Morse equivalent in the form:

$$
\Pi_{\mathrm{M}}(r)=-\epsilon\left\{1-\left[1-e^{-\alpha\left(r-r_{\mathrm{eq}}\right)}\right]^{2}\right\}
$$

where $r_{\text {eq }}$ defines the location of the minimum energy, and the $\alpha$ modifies the curvature of the potential energy at its minimum separation. The Morse potential is truncated with a third-order spline at the same cutoffs used in classical AIREBO as well as the splines for adaptive repulsion are implemented by the same technique. It was shown that the potential is capable of accurately modelling several hydrocarbons at very high pressures [25], but the potential still needs to be tested for large scale and more complex loading scenarios, such as hypervelocity impacts.

The purpose of this article is to report molecular dynamics simulation results on the hypervelocity dynamics of carbon systems at very high pressures, by employing the recently proposed 
AIREBO-M potential. We first consider shock-compressed graphite up to about $800 \mathrm{GPa}$. Among the various physical and chemical descriptions we focus here on the mechanical aspects, i.e. in the computation of material density, pressure, and shock velocity for accurate calculation of shock impedance of graphite. Comparisons with classical AIREBO [23] and LCBOPII [19] potentials are shown, along with various experimental and DFT data available in literature. We then perform equilibrium MD simulations to compute graphite thermal and mechanical properties to define an equation of state for continuum finite element model (FEM) of hypervelocity shock propagation in graphitic materials at the macroscale level. Second, we compare the results of MD impact simulation between AIREBO-M and its classical variant on graphene armours of variable number of layers impacted by an hypervelocity mass to study the dimensional scaling of the specific absorbed energy. Results indicate that this potential is a promising solution for the fast and accurate modelling, also multiscale, of the high-speed dynamics of graphene foams and graphene-based nanocomposites under hypervelocity shock compression as well as impact-/shock- induced dynamic fracture.

\section{Setup of computational models}

\subsection{Shock MD simulations}

To simulate shock-compression we built a 3D sample whose longitudinal dimension $(z)$ is significantly larger than the transverse sizes $(x, y$, see Figure 1). The minimum length of the sample along $z$ is chosen to allow the shock front to reach a stable planar front (also sufficiently far from the piston when Hugoniot is computed), to include a sufficient number of bins with enough atoms for accurate and high-resolution computation of the profile of shock state variables, and to account for induction time of the G/D transition in order to reach reaction equilibrium before the passage of the rarefaction shock wave reflected by the free edge. The initial supercell size is $V_{0}=3.86 \times 3.76 \times 99.18 \mathrm{~nm}^{3}$ including 170496 carbon atoms (resulting in 296 layers with 576 carbon atoms each). The atoms are assigned with initial velocities according to a Gaussian distribution corresponding to $T=600 \mathrm{~K}$, and thermal equilibration in the NPT ensemble is performed to a target temperature of $300 \mathrm{~K}$ and pressure of $0 \mathrm{MPa}$. The total equilibration time is $20 \mathrm{ps}$ with a timestep of $1 \mathrm{fs}$. We set periodic 
boundary conditions along all axes during equilibration obtaining a nominal bulk density of graphite $\rho_{0}=2.341 \mathrm{~g} / \mathrm{cm}^{3}$ (cell volume $3.87 \times 3.77 \times 99.45 \mathrm{~nm}^{3}$ ). For the shock simulation the periodic boundary condition along $z$ is removed, while remains along the other directions. The shock is generated by a rigid piston constituted by fixed atoms of two layers of graphene, and moved at a constant velocity $u_{\mathrm{p}}$ along the $z$ axis (Figure 1). The sampling bins for spatial average of quantities have thickness of $1.08 \mathrm{~nm}$ including the atoms of at least three graphene layers (1728 C particles) in the initial undeformed configuration. Shock simulations were then run in the microcanonical (NVE) ensemble with a time step of $0.1 \mathrm{fs}$ and for a time sufficiently large to let the shock wave to reach the free end of the sample at the opposite side of the piston. All simulations were performed with the package LAMMPS [26].

\subsection{Equilibrium thermodynamics simulations and FEM solution of the equation of state}

Continuum models require, together with continuity equations, the implementation of an equation of state (EOS) to determine the local state before and after the passage of the shock front. The acknowledged Grüneisen EOS [27] for compressed bulk material, assuming linear shock vs. particle velocity relationship, is defined as:

$$
p=\frac{\rho_{0} c_{0}^{2} \eta\left[1+\left(1-\frac{\gamma}{2}\right) \eta-\frac{\beta}{2} \eta^{2}\right]}{[1+(1-s) \eta]^{2}}+(\gamma+\beta \eta) e
$$

where $c_{0}$ and $s$ are the parameters determined from the linear fit of the shock vs. particle velocity Hugoniot (intercept and slope, respectively, see Results and discussion), $e$ is the specific internal energy per unit volume, $\eta=\rho / \rho_{0}-1=V_{0} / V-1, \beta$ is a first order dimensionless volume correction factor to $\gamma$, and $\gamma$ is the dimensionless Grüneisen parameter representing the change in pressure per energy variation at a constant volume, which can be expressed, in terms of thermodynamic, volumetric and mechanical properties, as [27]:

$$
\gamma=V\left(\frac{\partial p}{\partial e}\right)_{V}=\frac{\alpha K_{T}}{\rho c_{\mathrm{v}}}
$$

where $\alpha=\frac{1}{\Omega}\left(\frac{\partial \Omega}{\partial T}\right)_{p=0}$ is the volumetric thermal expansion coefficient $(\Omega=V / N$ is the atomic

volume), $K_{T}=-V_{\text {eq }}\left(\frac{\partial p}{\partial V}\right)_{T}$ is the isothermal bulk modulus ( $V_{\text {eq }}$ is the equilibrium volume determined from minimization of the potential energy of the system), and $c_{\mathrm{v}}=\left(\frac{\partial e}{\partial T}\right)_{V}$ is the 
specific heat capacity at constant volume. To derive $\gamma$ via Equation (4) a reduced cell of $3.93 \times 3.83 \times 4.09 \mathrm{~nm}^{3}$ with full periodic boundary conditions and containing 12 layers and 6912 atoms was used (nominal density of $2.367 \mathrm{~g} / \mathrm{cm}^{3}$ at $T=300 \mathrm{~K}$ after minimization). We verified that the cell is sufficiently large to avoid finite-size effect in the computation of the variables of interests. The range of temperatures explored lies between $400 \mathrm{~K}$ and $4000 \mathrm{~K}$ (the latter slightly below the expected melting/sublimation point of graphite [28, 29]) with step of $400 \mathrm{~K}$. To compute $\Omega$ and $\alpha$ we ran simulations with NPT ensemble for $20 \mathrm{ps}$ at zero pressure with timestep of $1 \mathrm{fs}$, with properties averaged after $10 \mathrm{ps}$. To compute $c_{\mathrm{v}}$ we calculate the variation of internal energy associated with temperature change (linear regression within the interval $T=400 \mathrm{~K}-4000 \mathrm{~K}$ ) for a defined constant volume (reference volume after NPT equilibration at $400 \mathrm{~K}$ ). To compute $K_{T}$ the volume of the cell is hydrostatically perturbed at different temperatures within the elastic regime (volumetric strain $\varepsilon_{\mathrm{V}}=0.997$ ) using the NVT ensemble and performing a linear regression of the $p-V$ curve.

To demonstrate the validity of the atomistically computed EOS and, consequently, the self-consistency between shock and thermodynamics simulations, we performed also a FEM solution of the Grüneisen EOS (Equation (3) ) with parameters derived from both far-fromequilibrium shock $\left(s\right.$ and $\left.c_{0}\right)$ and equilibrium thermodynamics $(\gamma)$ MD simulations ( $\beta$ assumed equal to 0). The system is represented by a unit cell with the same aspect-ratio the one employed in MD calculations, with $V_{0}=5 \times 5 \times 130 \mathrm{~mm}^{3}$ and $\rho_{0}=2.341 \mathrm{~g} / \mathrm{cm}^{3}$. Loading and boundary conditions follow the same setup of MD simulations.

\subsection{MD impact simulation on multilayer graphite armours}

Armours with different number of layers $(N=1,2,4,6)$ were studied by comparing their specific energy absorption capability defined as $K_{\text {abs }} / N \propto N^{\alpha}$ [1, 30], i.e. energy dissipated per layer (equivalent to normalization with respect to the target areal density). The targets are built as fully clamped circular layers with radius $R=9.8 \mathrm{~nm}$ while the impacting projectile is modeled as non-rigid diamond made of $2143 \mathrm{C}$ particles (van der Waals radius $r=1.43 \mathrm{~nm}$ ). For each case, the initial velocity $V_{0}$ assigned to the projectile corresponds to the minimum value necessary to have perforation of the target and, thus, a projectile residual velocity $V_{\text {res }}$ almost equal to zero. The absorbed energy $K_{\text {abs }}$ is computed from the difference in the centre 
of mass $(\mathrm{COM})$ translational velocity of the diamond projectile before and after perforation $\left(\Delta V=V_{0}-V_{\text {res }}\right)$ along the impact direction (i.e. normal to the target plane). The system is equilibrated using the NPT ensemble at $T=300 \mathrm{~K}$ before the impact stage, which is solved in the NVE ensemble.

\section{Results and discussion}

\subsection{Hugoniot EOS}

Figure 2 reports the different Hugoniot curves resulting from our simulations with AIREBO$\mathrm{M}$ along with the results reported in various experimental [7, 8, 10, 17] and computational [18, 31] studies. Variables of interest are computed at the time at which the steady state shock wave has propagated along the entire length of the sample. Pressure and temperature are computed as spatial average over the whole sample by excluding the atomic COM contribution to the kinetic energy. The shock wave velocity $U_{\mathrm{s}}$ is computed indirectly by the time that the shock front requires to reach the free edge of the composite and is consistent with the Hugoniot jump prediction $U_{\mathrm{s}}=p / \rho_{0} u_{\mathrm{p}}$. Figure 3 reports shock profiles (pressure and temperature), obtained from bin sampling, for a selection of computations together with snapshots of the same cases at full shock wave propagation with identification of the different crystalline structures within the samples.

Elastic compression is found for piston velocities up to about $2 \mathrm{~km} / \mathrm{s}$ (inset of Figure 2 a) for which the layered structure of graphite is preserved in the whole sample. From the fit of simulation points with the following well-known linear approximation of the velocity Hugoniot relationship:

$$
U_{\mathrm{s}}=s u_{\mathrm{p}}+c_{0}
$$

where $c_{0}=\sqrt{C_{11} / \rho_{0}}$ is the sound speed in the undeformed monodimensional elastic medium (as is nearly in our setup, being $C_{11}$ its Young's the modulus perpendicular to graphite planes) we determine $s=2.094, c_{0}=4.256 \mathrm{~km} / \mathrm{s}$ (linear regression in Figure 2.c). From $c_{0}$ we then derive $C_{11}=42.4 \mathrm{GPa}$, which is consistent with experimental measurements 32 and computation of elastic constants from equilibrium simulations on the reduced cell $\left(C_{11}=40.5 \mathrm{GPa}\right)$. Although 
a second or third order fit of the velocity Hugoniot could be used, it will be shown later that the linear EOS approximation (Equation (3)) is sufficiently accurate in the domain used for regression for the calculation of the $p-V$ Hugoniot via FEM continuum simulations.

The G/D transition was obtained for piston velocities ranging between 3 and $6 \mathrm{~km} / \mathrm{s}$ in accordance with the LCBOPII results by Pineau [18] and starting from pressure of about $50 \mathrm{GPa}$, consistently with the upper bound of experimental observations in literature [17, 32, 33]. The G/D transformation induces a visible inflection in both velocity and pressure Hugoniot. With respect to Pineau's work, where uniform transition to cubic diamond behind the shock front -with few local clusters of hexagonal diamond- was observed, we here obtain alternate stacking of cubic diamond -also accompanied by hexagonal diamond- and metastable graphite (Figure 3.c). This result is consistent with the experimental observations by Hirai and Kondo [34, which report a spacing between cubic phases between 0.07-0.2 nm after quenching.

The graphite/liquid transition is activated at piston velocities above $6 \mathrm{~km} / \mathrm{s}$ with a further increase in the slope of the $U_{\mathrm{s}}-u_{\mathrm{p}}$ curve. With respect to LCBOPII potential the shock velocity and pressure are lower, despite the slightly higher density in our work, and in closer agreement with the results by Nellis et al. [10], starting from moderate pressures (Figures 2.b and 6.b). Since the transverse sizes of the simulated cell are similar to Ref. [18], finite size effects are excluded and the different result, i.e. lower stiffness, can be imputed to the potential only. This may come out by the fact that the AIREBO-M potential, with respect to LCBOPII, was specifically designed to handle such extremely high pressures. On the contrary, the computed temperatures in the liquid phase (Figure 2,c) are higher than the LCBOPII, but to the best of the authors knowledge there is no data available to confirm these predictions. This may be imputed to the role of thermal electronic excitations which cannot be neglected and are not considered also in this interaction law. Such effects were quantified by Romero and Mattson [35] for shocked diamond, showing a significant decrease in the shock temperature by DFT simulations with finite electronic temperature.

Figure 4 finally shows a meaningful example of comparison between classical AIREBO and AIREBO-M depicting the pressure and temperature profiles, resulting from $u_{\mathrm{p}}=4 \mathrm{~km} / \mathrm{s}$ 
after $5 \mathrm{ps}$, together with snapshots of simulations. It is clear how the singularity of the LJ tail in the classical AIREBO affects the computation of interatomic repulsion forces, resulting in sensibly higher pressure, atomic and shock propagation velocities, and temperature, providing an unphysical behaviour (i.e. phase transition to amorphous carbon) even at relatively moderate piston velocities. We have verified that even a reduction of the timestep of one order of magnitude $(\Delta t=0.01 \mathrm{fs}$ ) is not sufficient to mitigate the problem (see Figure S1 in the Supplementary Material). Thus, the use of the classical variant should be carefully evaluated when modelling shock compression of carbon structures and possibly limited to equilibrium MD simulations. However, even at low pressure AIREBO-M has been proved to provide better results than the classical variant, since the Morse potential was fitted to reproduce the modulus and the c-axis spacing of graphite, without compromising the quality of AIREBO in the attractive range [25] and at nearly no additional computational cost. Thus, we expect similar results when modeling fracture in a single molecule (such as graphene sheet and organic molecule) as well as the elastic response of hydrocarbon polymer compounds.

\subsection{Thermodynamics properties and Grüneisen parameter}

Figure 5. a reports the atomic volume at the different investigated temperatures while Figure 5.b reports the volumetric thermal expansion coefficient. The potential shows monotonic increase of both $\Omega$ and $\alpha$ with $T$. The computation of $\Omega$ at $0 \mathrm{~K}$ is included to allow computation of $\alpha$ at $400 \mathrm{~K}$. The isochoric specific heat capacity $c_{\mathrm{v}}$ is determined to be $c_{\mathrm{v}} \approx 2.17 \mathrm{~J} / \mathrm{gK}$, which is is in good agreement with the theoretical prediction $c_{\mathrm{v}}=3 N_{\mathrm{a}} k_{\mathrm{b}} / m_{\mathrm{w}}=2.08 \mathrm{~J} / \mathrm{gK}$ (valid only much above $0 \mathrm{~K}$, where $N_{\mathrm{a}}$ is the Avogadro's number, $k_{\mathrm{b}}$ the Boltzmann's constant, and $m_{\mathrm{w}}$ the molecular weight). In Figure 5.c monotonic softening of the isothermal bulk modulus with increasing temperature is observed, with values consistent with literature [32].

Figure 5.d depicts the computed Grüneisen parameter $\gamma$ obtained by Equation (4) in the investigated temperature range. AIREBO-M provides a value of $\gamma$ with limited variation with temperature change and its average value estimated in the range $400 \mathrm{~K}-4000 \mathrm{~K}(\bar{\gamma}=0.56)$ is in very good agreement to measures reported in different experimental works [36 38]. Results from continuum FEM shock simulations are included in Figure 6 where we also report a magnification of the velocity and pressure Hugoniots of Figure 2 a-b in the range $u_{\mathrm{p}}<2 \mathrm{~km} / \mathrm{s}$. 
The obtained curve is in very good agreement with atomistic results confirming that the potential is able to determine a reliable value of the Grüneisen $\gamma$. Thus, the resulting EOS would allow to use AIREBO-M for consistent atomistic-continuum coupling for multiscale shock simulation of carbon-based materials.

\subsection{Scaling of impact properties of multilayer graphene armours}

Figure 7 shows the scaling of the specific absorbed energy and the corresponding best-fitted scaling exponent $\alpha$, obtained by the two different potentials. The adoption of AIREBO-M results for all cases in higher computed $K_{\text {abs }}$, ranging from about $+3 \%(N=1)$ to $+17 \%$ $(N=6)$ with respect to AIREBO. This increasing gap can be easily explained by the fact that the membrane-like behaviour of the single layer does not induce high contact pressures $\left(p_{z z}\right)$ : the effect of LJ singularity is limited in this case and the difference among the two potentials is not significant. As $N$ increases the contact pressure monotonically increases, both due to the transition to a bending/bulk dominated behaviour [1] and due to the higher critical velocity $V_{0}$ necessary to perforate the target; the region under the projectile contact locally resembles the shock compressed graphite scenario analyzed in the previous sections. In the end, these yield in an inversion of the scaling of the absorbed energy (change in sign of the scaling exponent $\alpha$ ) which contributes to explain the observed inconsistency among different works between MD and DFTB, in particular the systematically lower strength and toughness -and related suboptimal scaling- computed by MD [1, 21, 22]. It emerges, consistently with the above previous results on shock compression, that LJ interaction can yield to inaccurate results even at relatively moderate pressures, here $\leq 60 \mathrm{GPa}$ (Figure 8). Note that the slightly positive value of alpha obtained here with AIREBO-M ( $\alpha=0.006$, Figure 7) is consistent with the theoretical prediction in a previous work [1] corresponding to the ratio $R / t \approx 4.2$, where $t$ is the equivalent thickness of the single layer here conventionally assumed equal to $0.3415 \mathrm{~nm}$, also confirming the validity of this potential in modelling dynamic fracture and related energy dissipation. Figure 8 reports the snapshots of the performed impact simulations of the different tested cases. 


\section{Conclusions}

In this paper we have demonstrated the suitability of the recently proposed AIREBO-M potential to model graphite and multilayer graphene armours under hypervelocity shock compression and impact, respectively, showing its advantages over other widely employed laws for carbon in terms of accuracy. Regarding shock compressed graphite, results are in good agreement with experiments and DFT data in the elastic, G/D phase transformation and liquid regimes, up to about $0.8 \mathrm{TPa}$ of pressure. G/D transition and melting under shock are well predicted, also in accordance with the results by LCBOPII potential but, with respect to this, a more accurate stiffness response is obtained, especially in the highest pressure regime. As a consequence of this result, we have also shown that the classical AIREBO potential is responsible of inaccurate computation of impact properties, and of their dimensional scaling, in multilayer graphene armours with respect to DFT calculations. In this sight, similar attention should be posed when modelling of equivalent system made of other 2D materials, such as hexagonal boron nitride. Finally, an accurate computation of elastic and thermodynamic properties provides also a reliable estimate of the Grüneisen equation of state, opening to the possibility of integrated atomistic to continuum modelling for multiscale dynamic simulation of the hypervelocity shock dynamics of carbon materials and related nano-composites at reasonable computational time scales.

\section{Acknowledgements}

This study was supported by the National Research Foundation of Korea (NRF, no. 2016M3D1A1900038 and 2019R1A2C4070690). SS acknowledges financial support from BrainKorea21 Plus Postdoc Scholarship (NRF). NMP is supported by the European Commission under the Graphene Flagship Core 2 (WP14 "Composites", no. 785219) and the FET Proactive ("Neurofibers", no. 732344) and by the Italian Ministry of Education, University and Research (MIUR) under the "Departments of Excellence" grant L. 232/2016, the ARS01-01384-PROSCAN Grant and the PRIN-20177TTP3S Grant. 


\section{Author contributions}

SS and SHR designed the study. SS carried out the atomistic and FEM simulations. SS performed data extraction and visualization, and wrote the original draft. SS, SHR, KK, and NMP analysed and interpreted the data, and revised and edited the final manuscript. SHR supervised the study.

\section{Declaration of competing interest}

The authors declare that they have no conflicts of interest.

\section{Data availability}

The raw/processed data required to reproduce these findings cannot be shared at this time as the data also forms part of an ongoing study. Data will be made available on request.

\section{Copyright}

(C) 2019, Elsevier. This is the post-print version of: S. Signetti, K. Kang, N.M. Pugno, S. Ryu. Atomistic modelling of the hypervelocity dynamics of shockcompressed graphite and impacted graphene armours. Computational Materials Science, 170:109152, 2019. The publisher's version can be found at the following DOI: $10.1016 /$ j.commatsci.2019.109152. This version is released under the Creative Commons Attribution-Non Commercial-No Derivs 3.0 Unported (CC BY-NC-ND 3.0) License. 


\section{Figures}

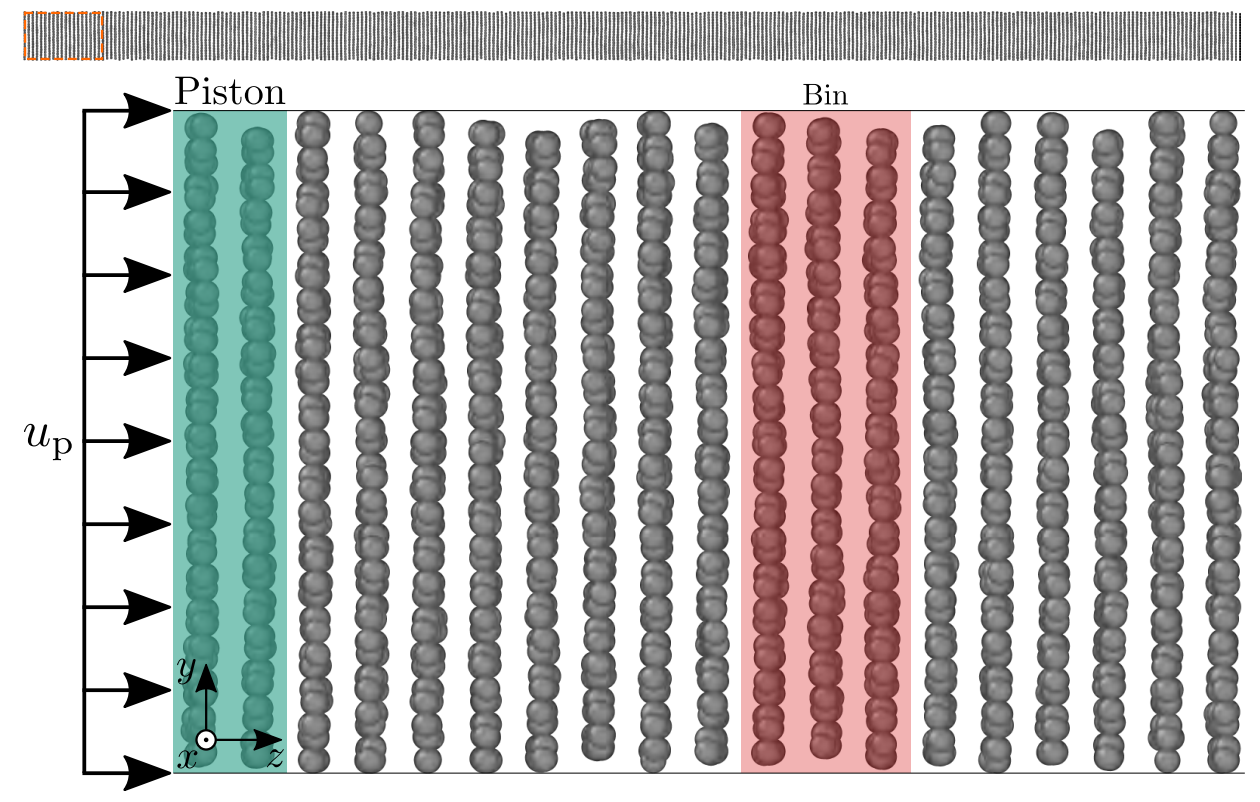

Figure 1: MD model setup for the shock simulations. The magnification shows a limited region of the whole graphite sample identified above. For thermodynamical characterization a shorter box containing 12 graphite layers is used. 
(a)

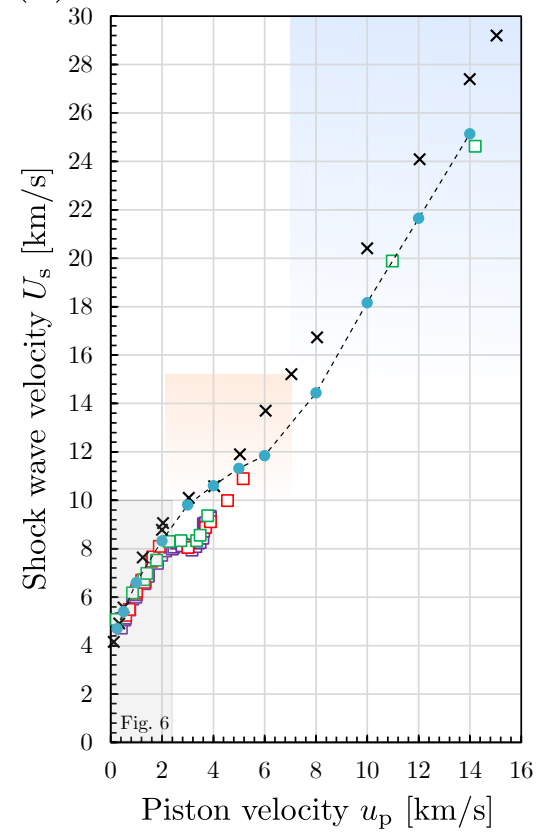

$\square$ LASL - PG $\square$ Gust - PG $\square$ Hanfland et al. (b)

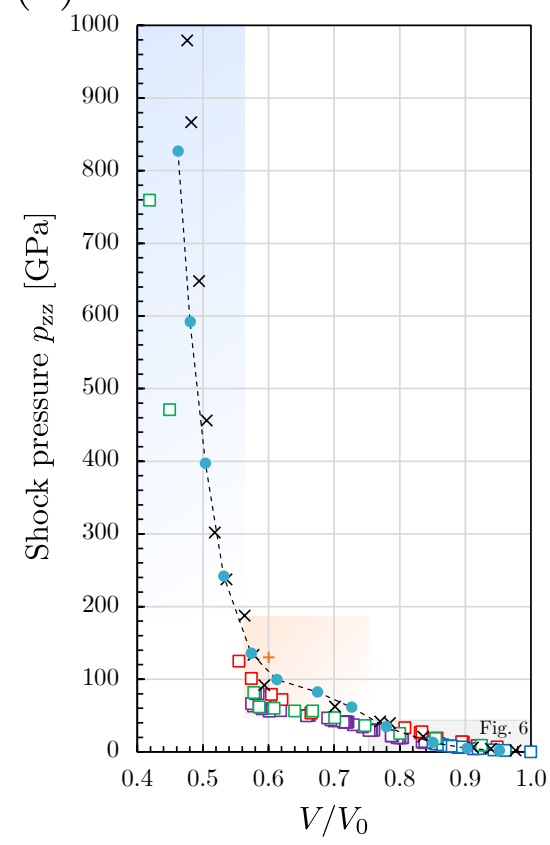

(c)

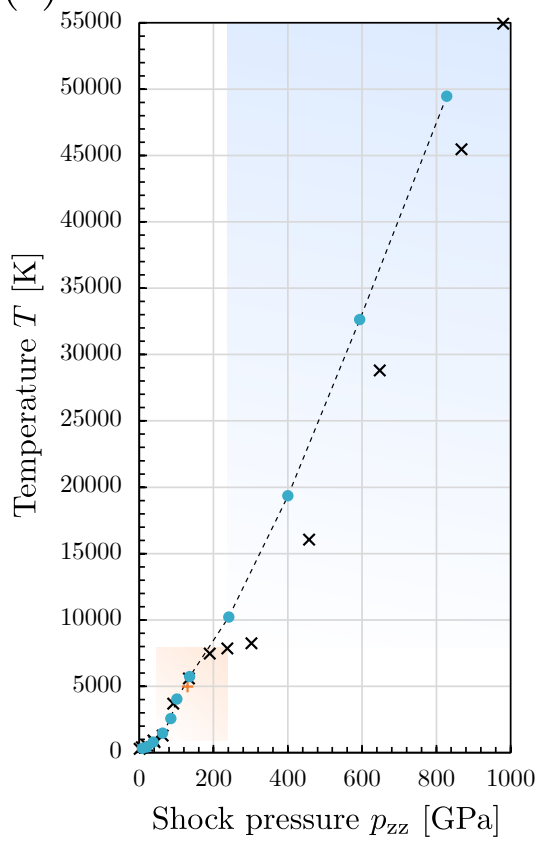

Figure 2: Hugoniot curves of shock compressed graphite $(\approx 8-900 \mathrm{GPa})$ obtained by MD simulations with AIREBO-M potential and compared with experimental data by Gust (pyrolytic graphite [8]), LASL Shock Hugoniot Data (pyrolytic graphite, $\rho_{0}=2.21 \mathrm{~g} / \mathrm{cm}^{3}$ [17]), Hanfland et al. [7], and Nellis et al. [10], DFT simulations by Mundy et al. [31, and MD simulations with LCBOPII potential by Pineau [18. Orange background highlights, qualitatively, the G/D transition domain, and the blue background highlights the region corresponding to liquid carbon. The dashed black curve is a guide to the eye to identify the Hugoniot curves obtained from the simulation points of this work. (a) $U_{\mathrm{S}}-u_{\mathrm{p}}$ velocity Hugoniot. (b) $p-V$ pressure Hugoniot. (c) $T-p$ temperature Hugoniot. For a magnification of the velocity and pressure Hugoniots in the range $u_{\mathrm{p}}<2 \mathrm{~km} / \mathrm{s}$ see Figure 6 a-b with additional results from FEM EOS simulations. 

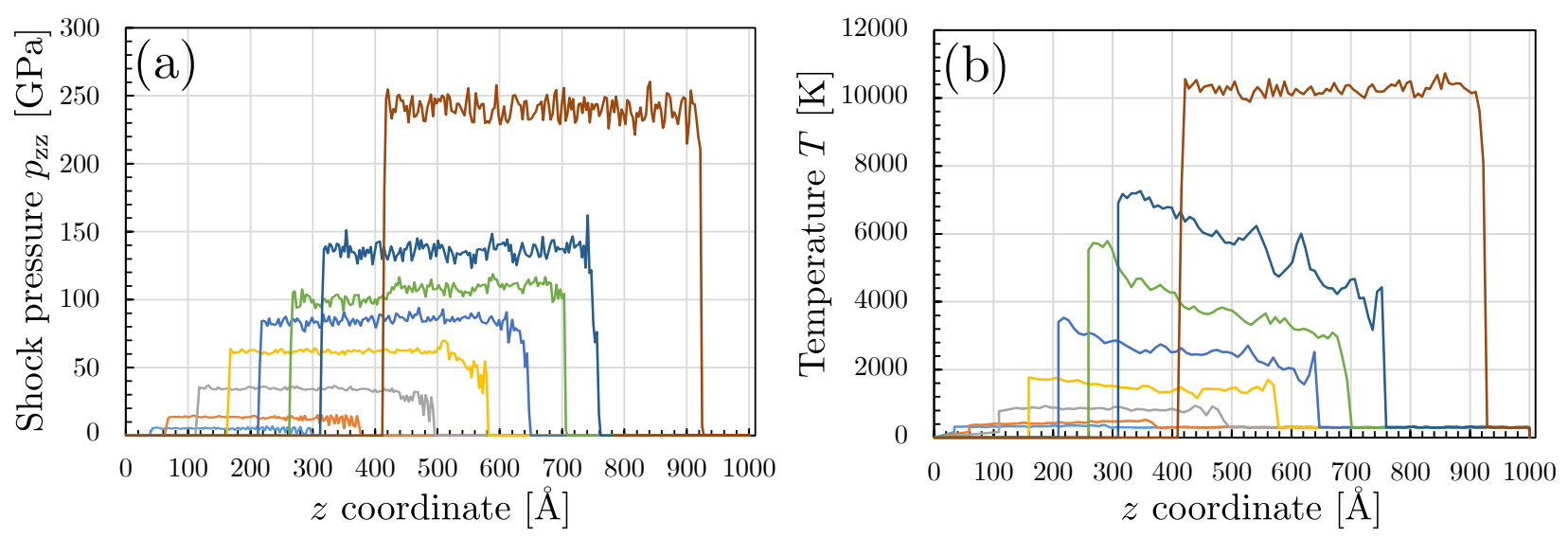

(c)

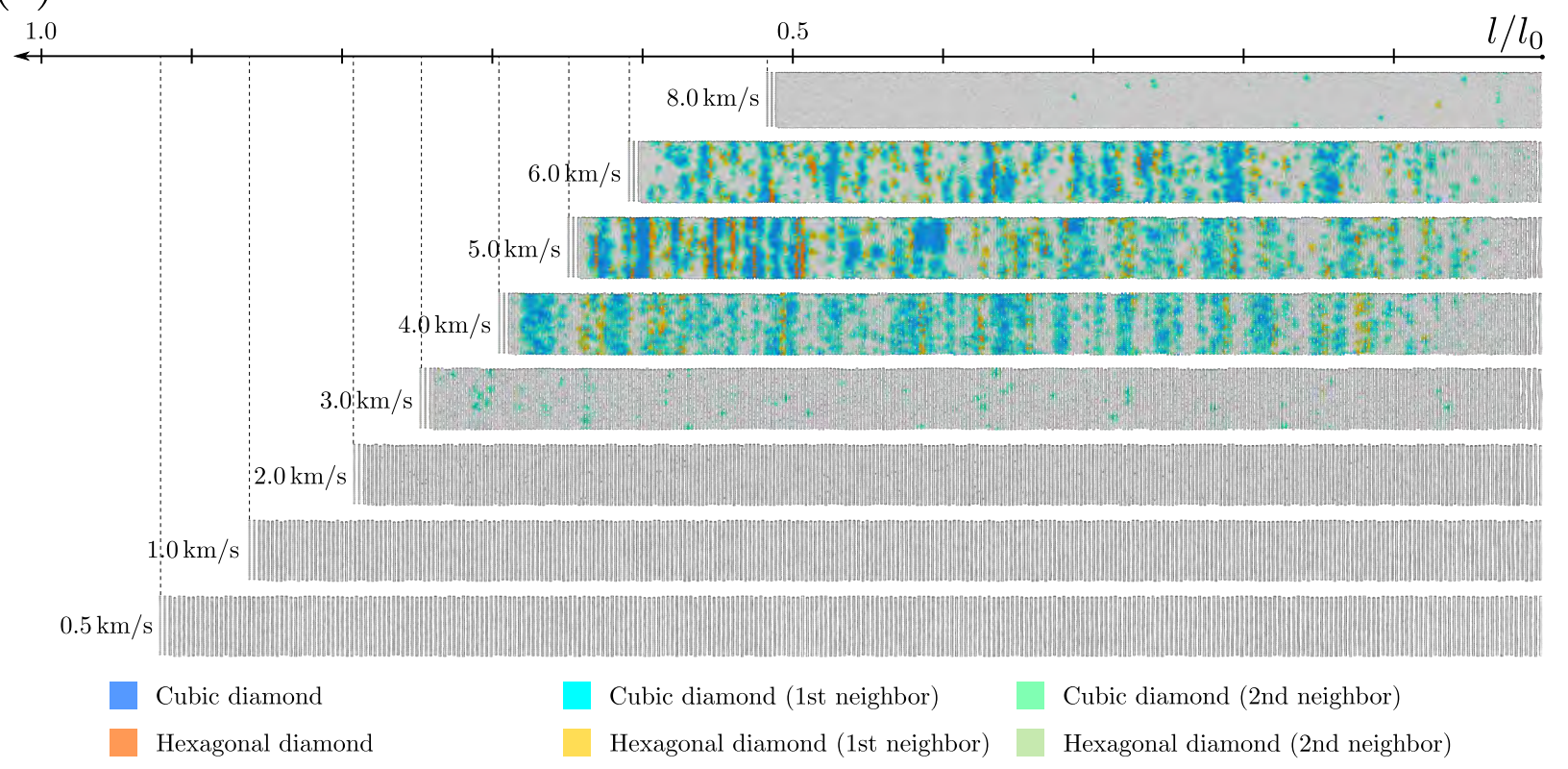

Figure 3: (a,b) Comparison of shock pressure and temperature profiles at $t=5 \mathrm{ps}$ for graphite shocked in the range $u_{\mathrm{p}}=0.5-8 \mathrm{~km} / \mathrm{s}$. (c) Snapshots of MD simulations $\left(u_{\mathrm{p}}=0.5-8 \mathrm{~km} / \mathrm{s}\right)$ taken at the corresponding time of propagation of the shock front through the whole sample length; the length of the sample after shock compression with respect to the original undeformed configuration $\left(l / l_{0}\right)$ is shown quantitatively on the graded axis. The different diamond structures forming within the sample due to shock are also shown (images elaborated with the visualization software OVITO [39]). 

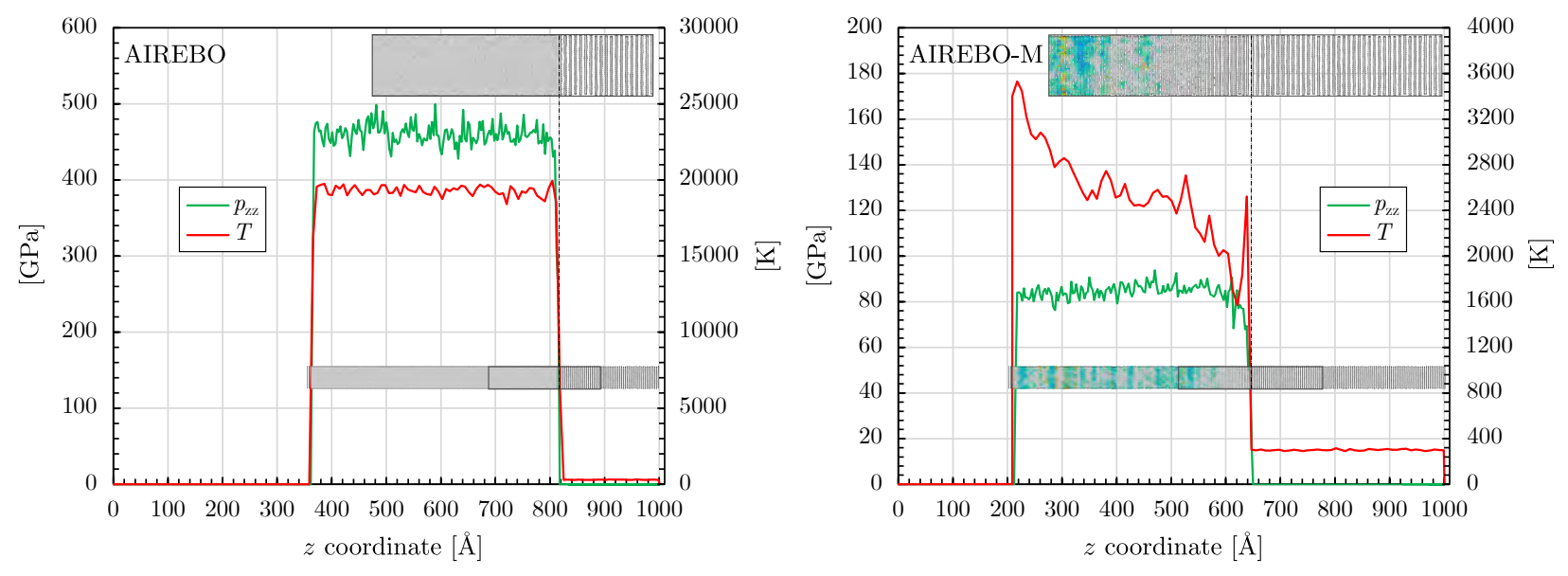

Figure 4: Comparison of shock pressure and temperature profiles obtained from MD simulations performed with classical AIREBO (left) and AIREBO-M right at $u_{\mathrm{p}}=4 \mathrm{~km} / \mathrm{s}(\Delta t=0.1 \mathrm{fs}$ ). Snapshots of the simulations and their magnification around the shock front (at $t=5 \mathrm{ps)}$ ) are superimposed to the diagrams (refer to legend of Figure 3 c for identification of diamond structures). See Figure S1 in the Supplementary Material for simulation with AIREBO and $\Delta t=0.01 \mathrm{fs}$. 

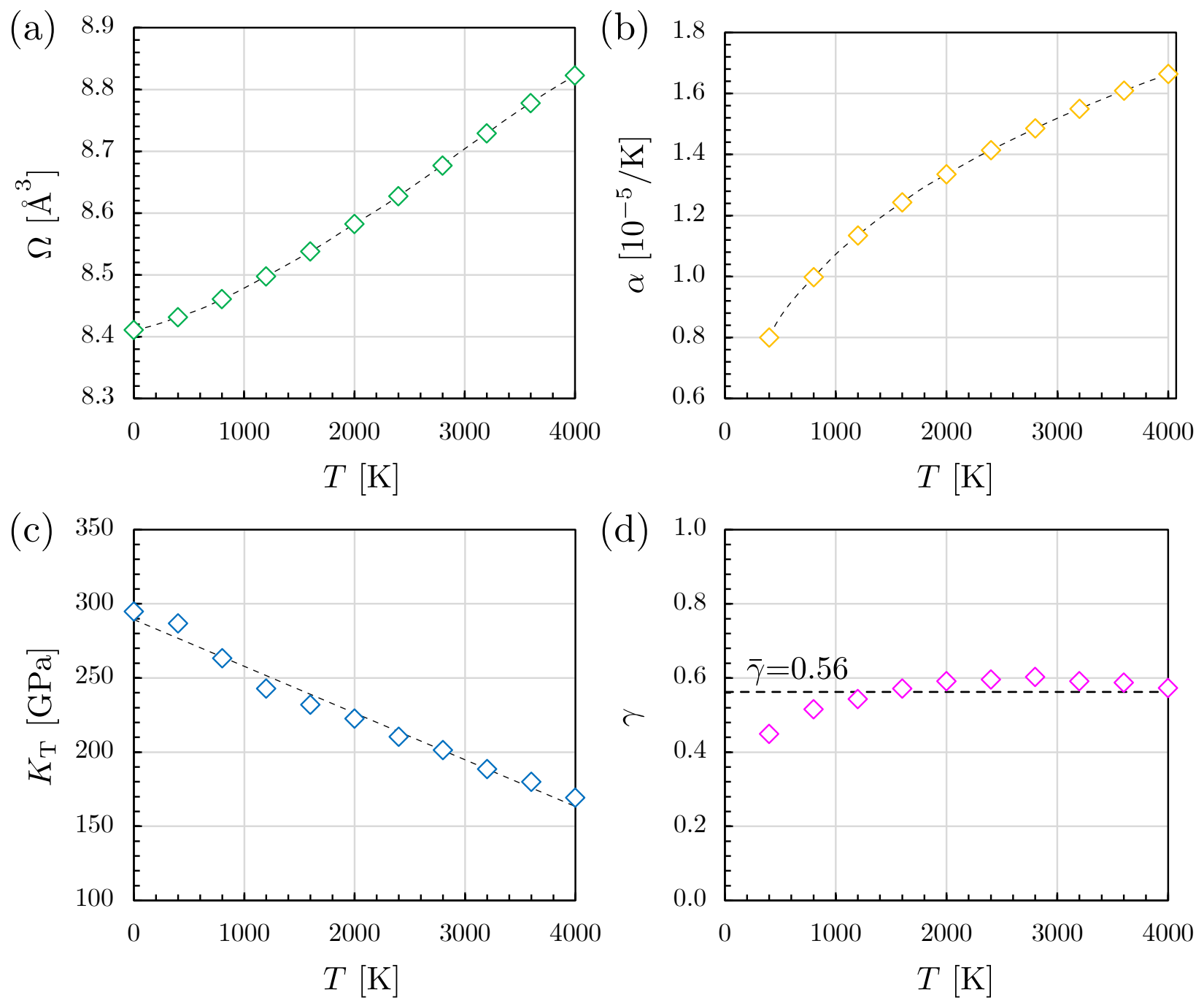

Figure 5: Volumetric, thermodynamic, and elastic properties of graphite determined from from MD simulations with AIREBO-M potential at different temperatures: (a) atomic volume, (b) volumetric thermal expansion coefficient, (c) isothermal bulk modulus, (d) Grüneisen parameter $\gamma$ (Equation (4)), the dashed line represents the average value, $\bar{\gamma}=0.56$, within the temperature range. 

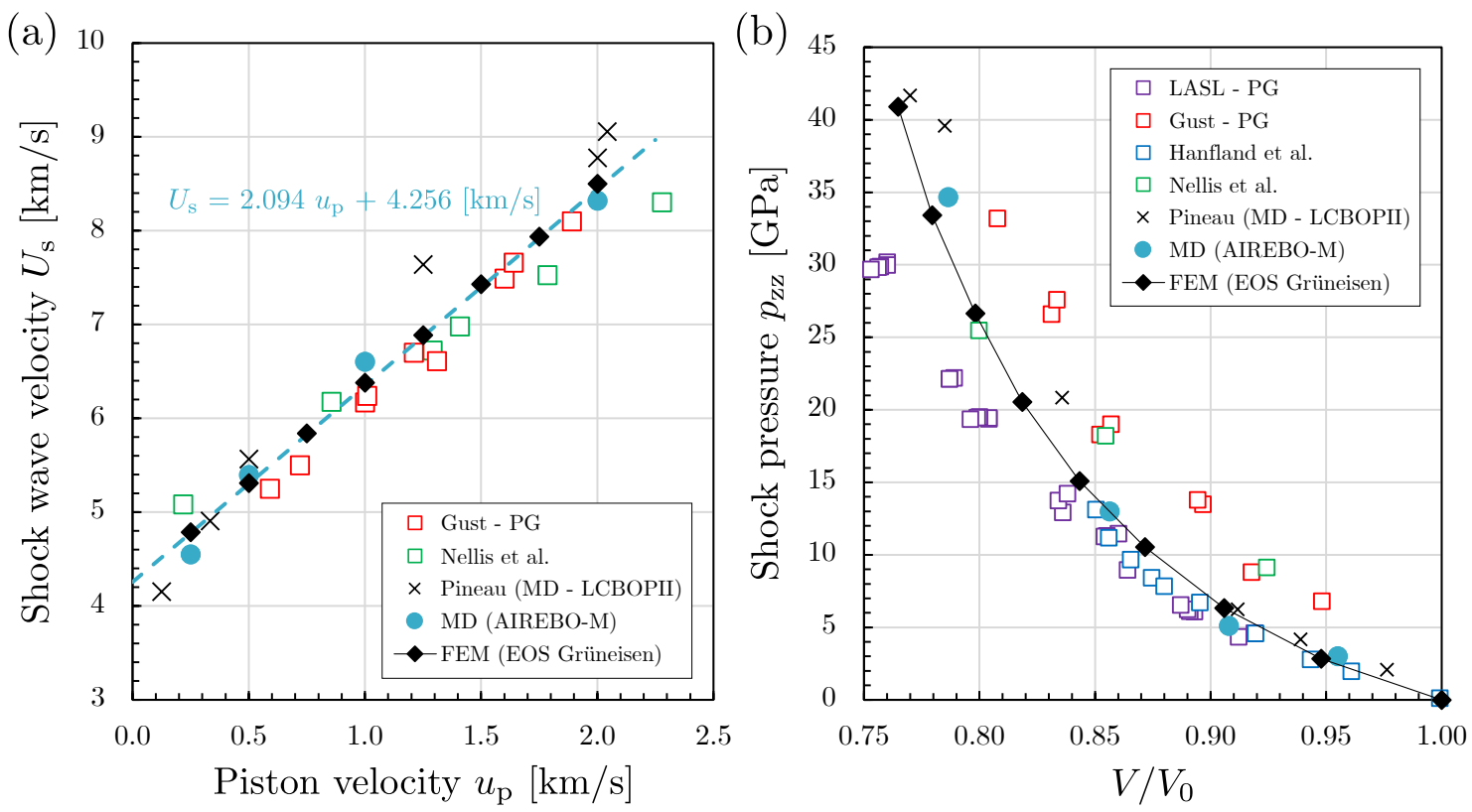

Figure 6: Magnified (a) velocity and (b) pressure Hugoniot of Figure 2 a-b in the range $u_{\mathrm{p}}<2 \mathrm{~km} / \mathrm{s}$ with addition of results from FEM simulation employing linear Grüneisen EOS (Equation (3p) with parameters derived from shock and equilibrium MD simulations using AIREBO-M (see Supplementary Video S1 for example of FEM shock simulation at $u_{\mathrm{p}}=500 \mathrm{~m} / \mathrm{s}$ ). The dashed line in panel (a) with related equation is the linear regression of MD points.

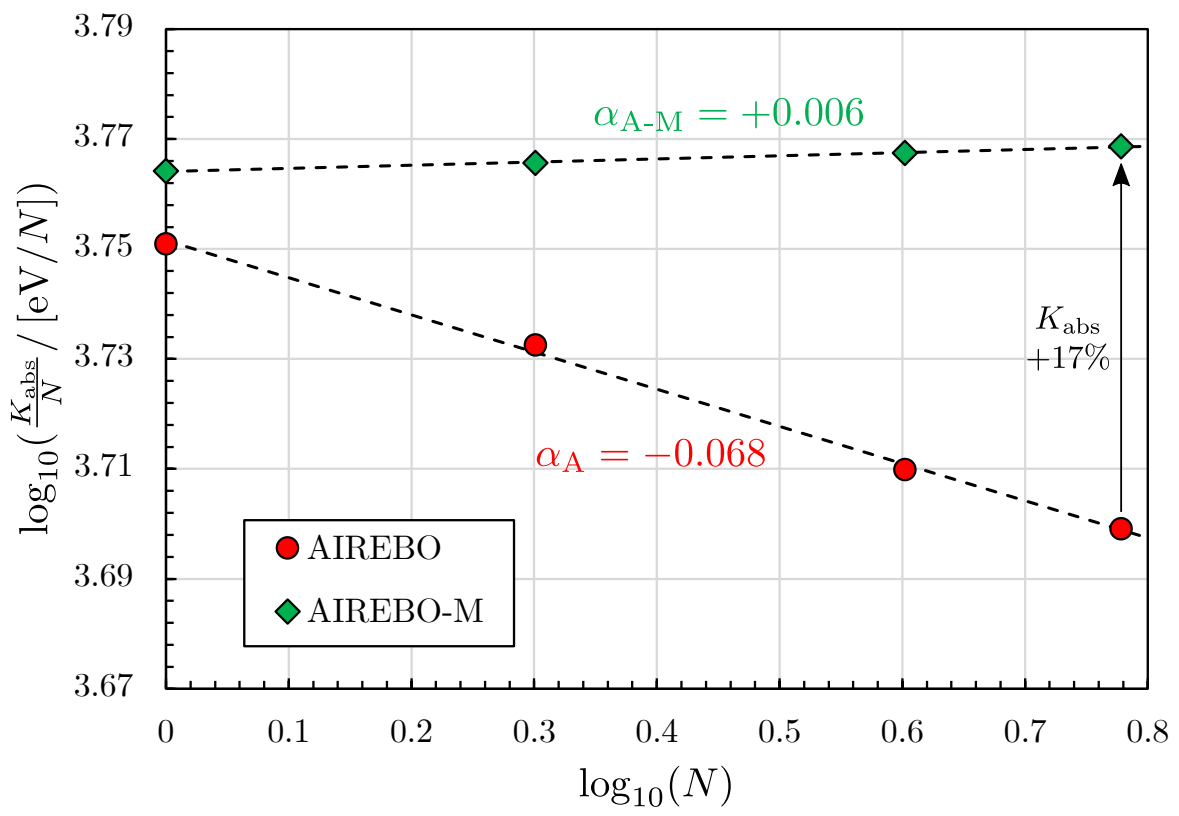

Figure 7: Scaling of specific absorbed energy in graphene armours under normal impact by a diamond spherical projectile by employing the two different AIREBO variants. 


\section{AIREBO AIREBO-M}
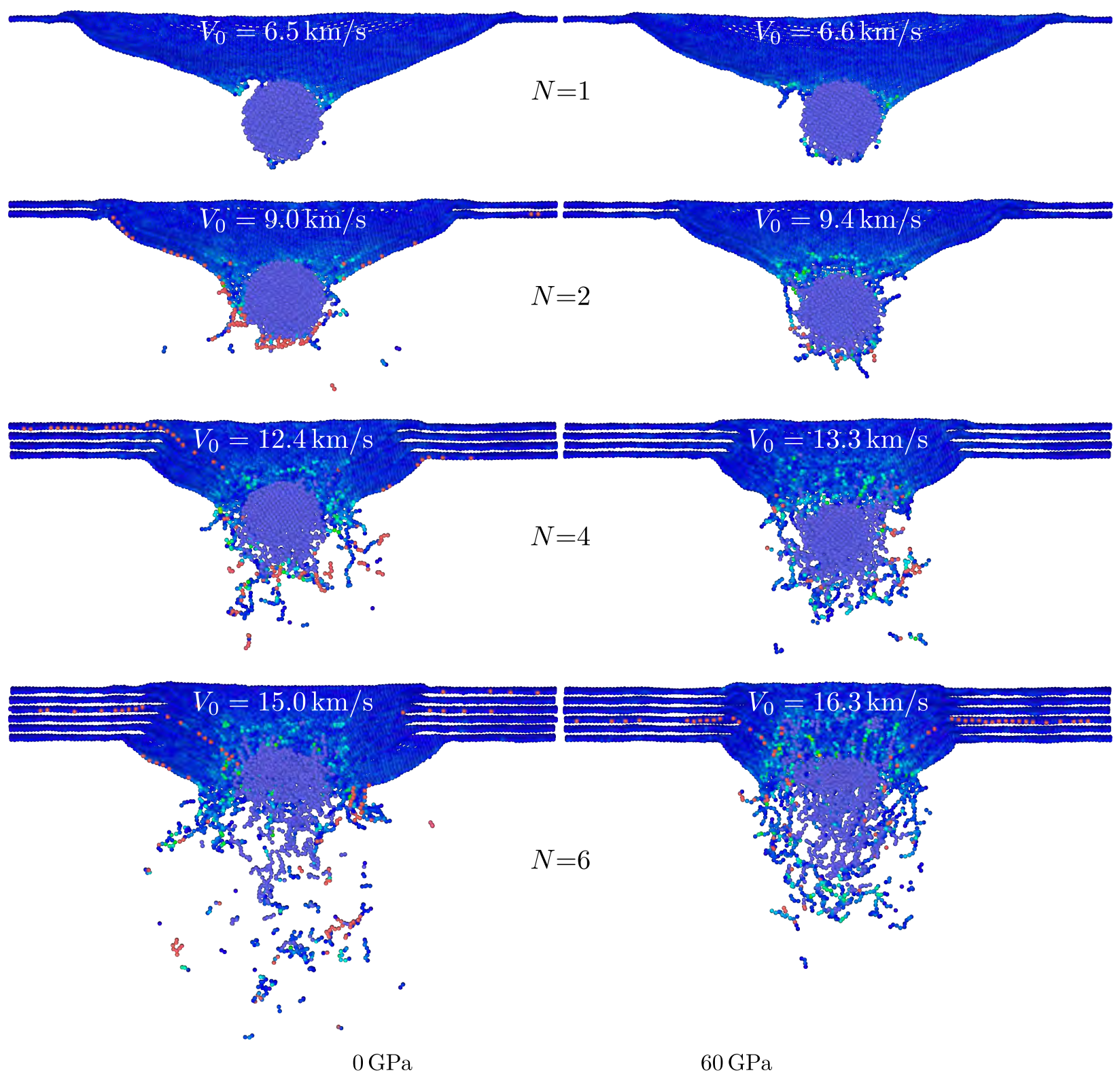

Figure 8: Snapshots of MD impact simulations on graphene armours with different number of layers $N$ obtained with AIREBO and AIREBO-M, showing contour of per-atom von-Mises stress. The corresponding impact velocity $V_{0}$ is the minimum required to allow full perforation of the targets for each case, for which the critical absorbed energy $K_{\text {abs }}$, reported in Figure 7 is calculated. 


\section{References}

[1] S. Signetti, S. Taioli, N. M. Pugno, 2D Material Armors Showing Superior Impact Strength of Few Layers, ACS Appl. Mater. Inter. 9 (46) (2017) 40820-40830. doi: 10.1021/acsami.7b12030.

[2] Y. Kim, J. Lee, M.-S. Yeom, W.-J. Shin, H. Kim, Y. Cui, J. Kysar, J. Hone, Y. Jung, S. Jeon, S.-M. Han, Strengthening effect of single-atomic-layer graphene in metal-graphene nanolayered composites, Nat. Commun. 4 (2013) 2114. doi: $10.1038 /$ ncomms 3114 .

[3] I. Gilmour, Geochemistry of carbon in terrestrial impact processes, Geol. Soc. of London, Special Publications 140 (1) (1998) 205-216. doi:10.1144/GSL.SP.1998.140.01.15.

[4] D. Kraus, A. Ravasio, M. Gauthier, D. O. Gericke, J. Vorberger, S. Fryfrych, J. Helfrich, L. B. Fletcher, G. Schaumann, B. Nagler, B. Barbrel, B. Bachmann, E. J. Gamboa, S. Göde, E. Granados, G. Gregori, H.-J. Lee, P. Neumayer, W. Schumaker, T. Döppner, R. W. Falcone, S. H. Glenzer, M. Roth, Nanosecond formation of diamond and lonsdaleite by shock compression of graphite, Nat. Commun. 7 (2016) 10970. doi:10.1038/ncomms 10970.

[5] G. F. Lindal, J. R. Lyons, D. N. Sweetnam, V. R. Eshleman, D. P. Hinson, G. L. Tyler, The atmosphere of Uranus: Results of radio occultation measurements with Voyager 2, J. of Geophys. Res.-Space 92 (A13) (1987) 14987-15001. doi:10.1029/JA092iA13p14987.

[6] W. B. Hubbard, Neptune's Deep Chemistry, Science 275 (5304) (1997) 1279-1280. doi:10.1126/science.275.5304.1279.

[7] M. Hanfland, H. Beister, K. Syassen, Graphite under pressure: Equation of state and first-order Raman modes, Phys. Rev. B 39 (17) (1989) 12598-12603. doi:10.1103/ PhysRevB.39.12598.

[8] W. H. Gust, Phase transition and shock-compression parameters to $120 \mathrm{GPa}$ for three 
types of graphite and for amorphous carbon, Phys. Rev. B 22 (10) (1980) 4744-4756. doi:10.1103/PhysRevB.22.4744.

[9] J. M. Winey, Y. M. Gupta, Shock-compressed graphite to diamond transformation on nanosecond time scales, Phys. Rev. B 87 (17) (2013) 174104. doi:10.1103/PhysRevB. 87.174104 .

[10] W. J. Nellis, A. C. Mitchell, A. K. McMahan, Carbon at pressures in the range 0.1-1 TPa (10 Mbar), J. Appl. Phys. 90 (2) (2001) 696-698. doi:10.1063/1.1381047.

[11] M. D. Knudson, M. P. Desjarlais, D. H. Dolan, Shock-Wave Exploration of the HighPressure Phases of Carbon, Science 322 (5909) (2008) 1822-1825. doi:10.1126/science. 1165278.

[12] D. K. Bradley, J. H. Eggert, D. G. Hicks, P. M. Celliers, S. J. Moon, R. C. Cauble, G. W. Collins, Shock Compressing Diamond to a Conducting Fluid, Phys. Rev. Lett. 93 (19) (2004) 195506. doi:10.1103/PhysRevLett.93.195506.

[13] H. Nagao, K. G. Nakamura, K. Kondo, N. Ozaki, K. Takamatsu, T. Ono, T. Shiota, D. Ichinose, K. A. Tanaka, K. Wakabayashi, K. Okada, M. Yoshida, M. Nakai, K. Nagai, K. Shigemori, T. Sakaiya, K. Otani, Hugoniot measurement of diamond under laser shock compression up to 2 TPa, Phys. Plasmas 13 (5) (2006) 052705. doi:10.1063/1.2205194.

[14] S. Brygoo, E. Henry, P. Loubeyre, J. Eggert, M. Koenig, B. Loupias, A. Benuzzi-Mounaix, M. R. L. Gloahec, Laser-shock compression of diamond and evidence of a negative-slope melting curve, Nat. Mat. 6 (2007) 274-277. doi:10.1038/nmat1863.

[15] D. G. Hicks, T. R. Boehly, P. M. Celliers, D. K. Bradley, J. H. Eggert, R. S. McWilliams, R. Jeanloz, G. W. Collins, High-precision measurements of the diamond Hugoniot in and above the melt region, Phys. Rev. B 78 (17) (2008) 174102. doi:10.1103/PhysRevB.78. 174102 .

[16] R. S. McWilliams, J. H. Eggert, D. G. Hicks, D. K. Bradley, P. M. Celliers, D. K. Spaulding, T. R. Boehly, G. W. Collins, R. Jeanloz, Strength effects in diamond under 
shock compression from 0.1 to 1 TPa, Phys. Rev. B 81 (1) (2010) 014111. doi:10.1103/ PhysRevB.81.014111.

[17] S. P. Marsh (Ed.), LASL Shock Hugoniot Data, Los Alamos Scientific Laboratory Series on Dynamic Material Properties, Vol. 5, University of California Press, 1980.

[18] N. Pineau, Molecular Dynamics Simulations of Shock Compressed Graphite, J. Phys. Chem. C 117 (24) (2013) 12778-12786. doi:10.1021/jp403568m.

[19] J. H. Los, L. M. Ghiringhelli, E. J. Meijer, A. Fasolino, Improved long-range reactive bond-order potential for carbon. I. Construction, Phys. Rev. B 72 (21) (2005) 214102. doi:10.1103/PhysRevB.72.214102.

[20] N. Pineau, L. Soulard, L. Colombet, T. Carrard, A. Pellé, P. Gillet, J. Clérouin, Molecular dynamics simulations of shock compressed heterogeneous materials. II. The graphite/diamond transition case for astrophysics applications, J. Appl. Phys. 117 (11) (1912) 115902. doi:10.1063/1.4914481.

[21] B. Z. G. Haque, C. S. C. Chowdhury, J. W. Gillespie, Molecular Simulations of Stress Wave Propagation and Perforation of Graphene Sheets Under Transverse Impact, Carbon 102 (2016) 126-140. doi:10.1016/j.carbon.2016.02.033.

[22] K. Yoon, A. Ostadhossein, A. D. T. van Duin, Atomistic-scale Simulations of the Chemomechanical Behavior of Graphene under Nanoprojectile Impact, Carbon 99 (2016) 58-64. doi:10.1016/j.carbon.2015.11.052.

[23] S. J. Stuart, A. B. Tutein, J. A. Harrison, A reactive potential for hydrocarbons with intermolecular interactions, J. Chem. Phys. 112 (14) (2000) 6472-6486. doi:10.1063/1 481208 .

[24] D. W. Brenner, O. A. Shenderova, J. A. Harrison, S. J. Stuart, B. Ni, S. B. Sinnott, A second-generation reactive empirical bond order (REBO) potential energy expression for hydrocarbons, J. Phys-Condens. Mat. 14 (4) (2002) 783. doi:10.1088/0953-8984/14/ $4 / 312$. 
[25] T. C. O'Connor, J. Andzelm, M. O. Robbins, AIREBO-M: A reactive model for hydrocarbons at extreme pressures, J. Chem. Phys. 142 (2) (2015) 024903. doi: $10.1063 / 1.4905549$.

[26] S. Plimpton, Fast Parallel Algorithms for Short-Range Molecular Dynamics, J. Computat. Phys. 117 (1) (1995) 1-19. doi:10.1006/jcph.1995.1039.

[27] E. Grüneisen, Theorie des festen Zustandes einatomiger Elemente, Ann. Phys. 344 (12) (1912) 257-306. doi:10.1002/andp.19123441202.

[28] L. M. Ghiringhelli, J. H. Los, E. J. Meijer, A. Fasolino, D. Frenkel, Modeling the Phase Diagram of Carbon, Phys. Rev. Lett. 94 (14) (2005) 145701. doi:10.1103/PhysRevLett. 94.145701.

[29] L. X. Benedict, K. P. Driver, H. Sebastien, B. Militzer, T. Qi, A. A. Correa, A. Saul, E. Schwegler, Multiphase equation of state for carbon addressing high pressures and temperatures, Phys. Rev. B 22 (2014) 224109. doi:10.1103/PhysRevB.89.224109.

[30] S. Signetti, N. M. Pugno, Evidence of optimal interfaces in bio-inspired ceramic-composite panels for superior ballistic protection, J. Eur. Ceram. Soc. 34 (11) (2014) 2823-2831. doi:10.1016/j.jeurceramsoc.2013.12.039.

[31] J. C. Mundy, A. Curioni, N. Goldman, I. F. W. Kuo, E. J. Reed, L. E. Fried, M. Ianuzzi, Ultrafast transformation of graphite to diamond: An ab initio study of graphite under shock compression, J. Chem. Phys. 128 (18) (2008) 184701. doi:10.1063/1.2913201.

[32] F. J. Ribeiro, P. Tangney, S. G. Louie, M. L. Cohen, Structural and electronic properties of carbon in hybrid diamond-graphite structures, Phys. Rev. B 72 (2005) 214109. doi: 10.1103/PhysRevB.72.214109.

[33] S. Scandolo, M. Bernasconi, G. L. Chiarotti, P. Focher, E. Tosatti, Pressure-Induced Transformation Path of Graphite to Diamond, Phys. Rev. Lett. 74 (20) (1995) 4015-4018. doi:10.1103/PhysRevLett.74.4015. 
[34] H. Hirai, K.-I. Kondo, Modified Phases of Diamond Formed Under Shock Compression and Rapid Quenching, Science 253 (5021) (1991) 772-774. doi:10.1126/science.253. 5021.772 .

[35] N. A. Romero, W. D. Mattson, Density-Functional Calculation of the Shock Hugoniot for Diamond, Phys. Rev. B 76 (2007) 214113. doi:10.1063/1.4914481.

[36] W. B. Gauster, Elastic constants and grüneisen parameters of pyrolytic graphite, Philos. Mag. 25 (3) (1972) 687-700. doi:10.1080/14786437208228900.

[37] D. A. Benson, W. B. Gauster, Grüneisen parameters of pyrolytic graphites, Philos. Mag. 31 (5) (1975) 1209-1222. doi:10.1080/00318087508226839.

[38] A. P. Stepovik, The Grüneisen coefficient for UPV-1 pyrolytic carbon, J. Appl. Mech. Tech. Phy. 33 (4) (1992) 611-613. doi:10.1007/BF00864291.

[39] A. Stukowski, Visualization and analysis of atomistic simulation data with OVITO - the Open Visualization Tool, Model. Simul. Mater. Sc. 18 (2010) 015012. doi:10.1103/ PhysRevB .89.224109. 


\title{
Atomistic modelling of the hypervelocity
}

\section{dynamics of shock-compressed graphite and}

\author{
impacted graphene armours
}

\section{- Supplementary Material -}

Stefano Signetti, ${ }^{\dagger}$ Keonwook Kang, ${ }^{*, \dagger}$ Nicola Pugno, $, \AA, \S, \|$ and Seunghwa Ryu ${ }^{*, \dagger}$

$\dagger$ Department of Mechanical Engineering,

Korea Advanced Institute of Science and Technology (KAIST),

291 Daehak-ro, Yuseong-gu, Daejeon 34141, Republic of Korea

$\ddagger$ Department of Mechanical Engineering, Yonsei University,

50 Yonsei-ro, Sodaemun-gu, Seoul 03722, Republic of Korea

\Laboratory of Bio-Inspired and Graphene Nanomechanics,

Department of Civil, Environmental and Mechanical Engineering, University of Trento, via Mesiano 77, I-38123 Trento, Italy.

$\S$ School of Engineering and Materials Science, Queen Mary University of London, E1 4 NS London, UK

|| Ket-Lab, Edoardo Amaldi Foundation, I-00133 Roma, Italy

E-mail: kwkang@yonsei.ac.kr; ryush@kaist.ac.kr 


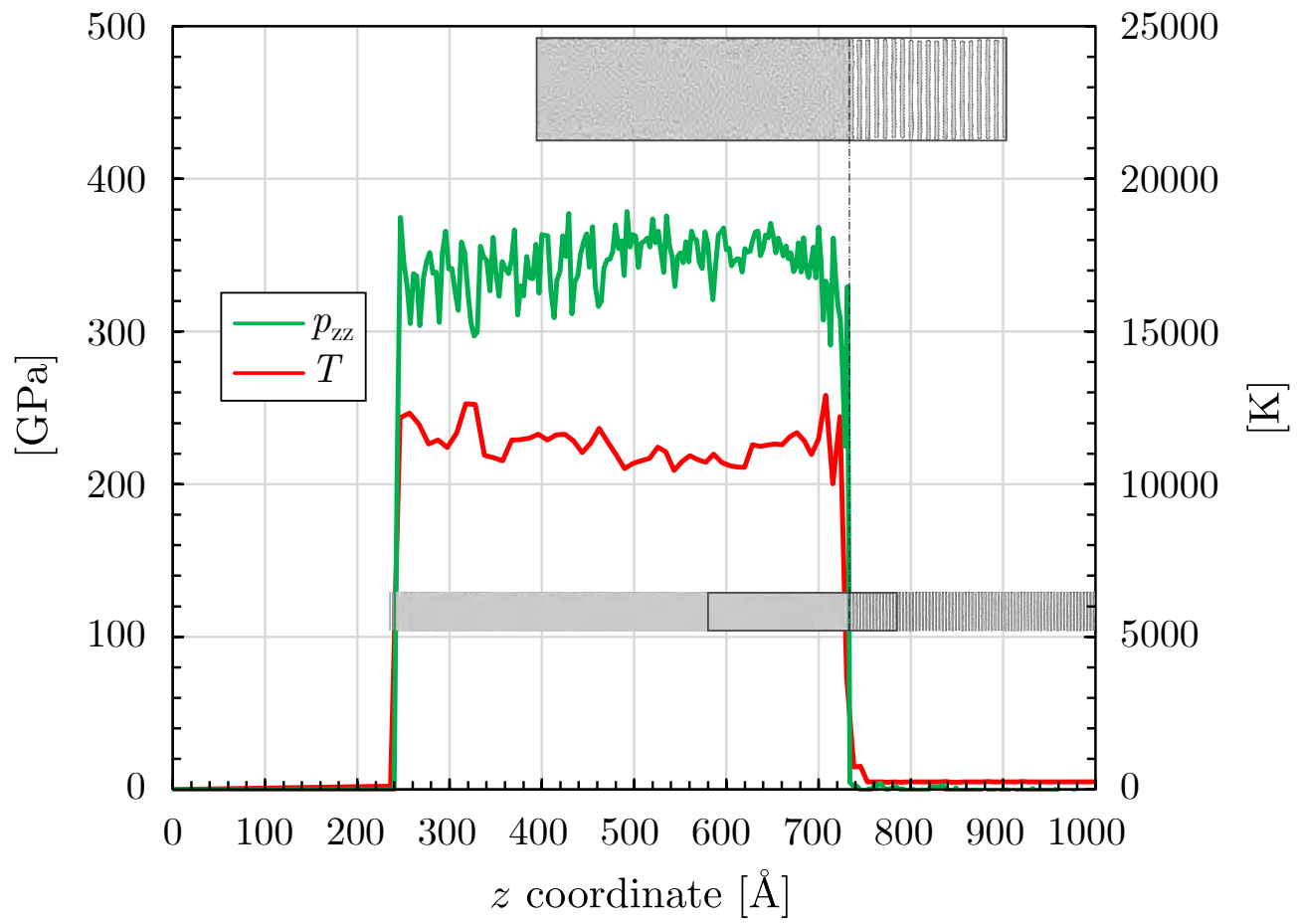

Figure S1: Shock pressure and temperature profiles obtained from MD simulations performed with classical AIREBO at $u_{\mathrm{p}}=4 \mathrm{~km} / \mathrm{s}(\Delta t=0.01 \mathrm{fs})$. Snapshots of the simulation and its magnification around the shock front (at $t=5 \mathrm{ps)}$ ) are superimposed to the diagrams (see Figure 4 .a in the main text for comparison with simulation using $\Delta t=0.1 \mathrm{fs}$ ).

\section{Supplementary videos}

Video S1. FEM shock simulation on graphite sample at $u_{\mathrm{p}}=500 \mathrm{~m} / \mathrm{s}$. Video-S1.mp4 